\title{
Photovoltage and photocurrent spectroscopy of luminescent porous silicon
}

\author{
O.V. Vakulenko, S.V. Kondratenko \\ National Taras Shevchenko Univiversity of Kyiv, 6 Glushkova Ave., 03022 Kyiv, Ukraine \\ e-mail:kondr@univ.kiev.ua
}

\begin{abstract}
Measurements of photoconductivity, photovoltage and photoluminescence spectra of porous silicon/c-Si structures are carried out. It is shown that the shape of the photoconductivity and photovoltage spectra is caused mainly by bulk properties of porous silicon. Two components of the photovoltage spectral dependence caused by porous silicon are observed. On the contrary, the one photoconductivity component is revealed. Moreover, the photoconductivity spectrum shape is found to depend on the value of the bias voltage and differ from the photovoltage spectrum in the short-wave region. The obtained results are explained within the model where the por-Si is considered as composite system of crystallites embedded in amourphous matrix of silicon compounds.
\end{abstract}

Keywords: photoconductivity, photovoltage, porous silicon.

Paper received 09.04.03; accepted for publication 16.06.03.

\section{Introduction}

The detection of intense photoluminescence in porous silicon (por-Si) [1] has attracted a great attention due to prospects of designing the effective photosensitive and electroluminescent devices as well as the different types of sensors based on this material [2-5]. The main efforts of scientists were applied to studying the luminescent, optical and structural properties of porous silicon [6-9]. A great amount of models have been proposed to explain the mechanism of visible photoluminescence in por-Si. Unfortunately, each of them explains only the limited number of experimental data. The peculiarities of nonequilibrium charge carrier transport in porous silicon associated with its non-uniformity and existence of crystalline and amorphous phases are not adequately investigated. At the same time, the study of such photoelectric properties as photoconductivity, photovoltage (PV) and photomagnetic effect has not received sufficient attention as compared with the luminescent and optical ones. Particularly, the research of mentioned photoelectric properties is power tool for determination of energy band structure of semiconductors and study of nonequilibrium charge carrier transport.
The photoconductivity and photovoltage in por-Si/cSi structures were studied in [2,3,10-14]. Measurements of photoconductivity [15] and surface photovoltage [16] spectra in free-standing por-Si films have been also carried out. Nevertheless, the non-uniformity of the porous silicon, particularly the presence of direction along which the physical properties change most considerably, has not been taken into account in the majority of works dedicated to analysis of nonequilibrium processes in por-Si. It is common knowledge that such direction is perpendicular to por-Si/c-Si interface and it is produced by gradient of crystallite size [17-18], mechanical stresses [19$20]$ and content of amorphous phase [12,21].

Presumably, the presence of mentioned gradients causes the non-uniformity of the por-Si band structure and the existence of the inner electric field. The possible existence of the inner electric field in the porous silicon bulk was assumed by the authors [21,22]. In the work [23], from analysis of the photomagnetic effect spectra in porous silicon it was found that the nonequilibrium charge carrier transport originates from both diffusion and drift in the inner electric field mainly in the direction perpendicular to the por-Si/c-Si interface.

One would expect both non-uniformity of the por-Si physical properties and existence of the inner electric 


\section{O.V. Vakulenko, S.V. Kondratenko: Photovoltage and photocurrent spectroscopy of ...}

field may become apparent in studying the photoconductivity and photovoltage spectra. Besides, the comparison of the photoconductivity, photovoltage and photoluminescence spectra can provide an additional information on the nonequilibrium processes in porous silicon.

The aim of this work is to elucidate the physical reasons which cause the shape of the photoconductivity and photovoltage spectra in porous silicon and to clear up the influence of its non-uniformity on nonequilibrium processes.

\section{Experiment}

The por-Si/c-Si structures were formed by anodization of p-type (111) crystalline silicon wafers with a resistivity of $10 \Omega \cdot \mathrm{cm}$ in $48 \mathrm{wt} \% \mathrm{HF}$ solution with current density $100 \mathrm{~mA} / \mathrm{cm}^{2}$ during 3 minutes. Before anodization, the wafers were cleaned, an ohmic contact was formed by a thin Al film onto the reverse side. The por-Si thickness was $25 \mathrm{~mm}$. After anodization an Al rectangular frame with the sizes of $5 \times 10 \mathrm{~mm}^{2}$ and the stripe thickness of 0.4 $\mathrm{mm}$ was evaporated onto por-Si surface (inset in Fig.1).

Measurements of the photoconductivity and photovoltage spectra were carried out at the temperature $293 \mathrm{~K}$ under illumination of the porous silicon layer. In doing so, the excited illumination was focused inside the Al frame in that way the metal was not illuminated. The chosen geometry made it possible, firstly, to decrease essentially the influence of the metal/ por-Si barrier height change with illumination which is negative for studying of por-Si bulk properties, and secondly to investigate the photoelectric properties of por-Si layers after rather long (one year) storage in the atmospheric environment. The gradient of crystallite sizes is maximum for such kind of structures [24]. The theoretical calculation described in [24] have shown that a smooth reduction of crystallites sizes is necessary in order to obtain an intense emission from the porous silicon structure. Moreover, the technology of contact formation by vacuum evaporation onto the por-Si surface immediately after anodization prevents from oxidation, hydrogen effusion, etc. to which the luminescent, optical and, probably, photoelectric por-Si properties are especially sensitive [25].

A $100 \mathrm{~W}$ halogen lamp served as a source of light, which provided the value of the excitation intensity of about $10^{14}$ quanta/sm ${ }^{2}$ Ys. Measurements of the photoconductivity were carried out at following values of reverse bias voltage: $U=1.5 ; 3$ and $4.5 \mathrm{~V}$. In the reverse direction the negative output of the external power supply was connected to c-Si substrate, and the positive output to por-Si surface. In addition the linear dependence of the photoconductivity and photovoltage signals on the excitation intensity took place in the all spectral region, which enabled us to normalize the obtained spectra to the constant number of the excitation quanta.

\section{Results and discussion}

It is well known that the photovoltaic effect (except Dumber effect) occurs in a semiconductor structure with built-in electric field caused by gradient of either potential or band gap width in certain direction. The electronhole pairs generated by photon absorption can be separated by such field and produce measurable potential drop across the sample.

In Fig. 1 a typical spectral dependence of the photovoltage in the por-Si/c-Si structure is shown. The shape of obtained spectral dependence is caused by contribution of tree components. In the long-wave region $(h v<1.3 \mathrm{eV})$, where the por-Si layer is transparent, the excitation radiation is adsorbed mainly in the c-Si substrate and one component only is observed. The photovoltage in this region is caused by separation of the nonequilibrium electron-hole pairs in the spatial charge field of the por-Si/c-Si heterojunction [22,23,25-27].

Further increase of excitation quantum energy resulted in the photovoltage growth. It can be explained by separation of nonequilibrium electron-hole pairs in the por-Si bulk. Moreover, the shape of PV spectrum is determined mainly by energy band structure of porous silicon when the excitation quantum energy exceeds $1.3 \mathrm{eV}$. The similar photovoltage spectra were observed for freestanding por-Si films. The only dissimilarity from PV spectra of por-Si/c-Si structure is an absence of long-wave PV component caused by electron-hole separation in the electric field of por-Si/c-Si heterojunction.

The typical "window effect" should be observed when the photovoltage is caused only by separation of nonequilibrium charge carriers in the spatial charge field of a heterojunction. This effect implies that a spectral sensitivity region is a band in the range of photon energy between the energy gaps for narrow-band and wide-gap materials $[13,28]$. Such shape of the spectral dependence is explained by decreasing the number of quanta adsorbed in the spatial charge region of the heterojunction. At that, the increase of absorption in the bulk of wide-gap material leads to photovoltage value drop. "Window effect" has been observed by the authors [13] in por-Si/c-Si structures with a thin (about $1 \mu \mathrm{m}$ ) porous silicon layer. In our case, an increase of PV value is observed while the absorption in porous silicon is increased, so the contribution of PV component which is generated in porous silicon bulk and is not due to the separation of the nonequilibrium charge carriers in the spatial charge layers of por-Si/c-Si heterojunction, observes in short-wave range. The relative contribution of this component is increased while the contribution caused by heterojunction is accordingly decreased at the sacrifice of increasing absorption in por-Si.

So, the shape of PV spectra in the region $h v>1.3 \mathrm{eV}$ is caused mainly by porous silicon bulk properties, i.e. electronic structure of studied samples. In the bulk materials the photovoltage is produced by separation of nonequilibrium electron-hole pairs in built-in electric 


\section{O.V. Vakulenko, S.V. Kondratenko: Photovoltage and photocurrent spectroscopy of ...}

field. Generally, such field caused by gradient of either potential or band gap width in certain direction. The physical properties of por-Si change most considerably in the direction perpendicular to the por-Si /c-Si interface [17-21]. Specially, it was found that the crystallite sizes decrease as they move away from por-Si/c-Si interface while the content of amorphous phase on the contrary increase $[17,18,21]$. Assumption of por-Si spatial non-uniformity is confirmed by continuos decrease of the photoluminescence band intensity as a function of distance from the top surface [29]. Therefore, the porous silicon can be considered as composite system of silicon crystallites in amorphous $\mathrm{SiO}_{\mathrm{x}} \mathrm{H}_{\mathrm{y}}$ matrix of silicon compounds with oxygen and hydrogen. At that, the near-surface region is amorphous with impregnation of practically isolated crystallites. On the contrary, near c-Si substrate the content of amorphous phase is minimal (or practically absent) and silicon crystallites have the largest size. Furthermore, the band gap width of nanoscale silicon crystallites depends on size due to quantum-size effect [1], while the band gap of silicon compounds $\mathrm{SiO}_{\mathrm{x}} \mathrm{H}_{\mathrm{y}}$ is determined by content of oxygen and hydrogen [21]. As a results, the band gap of por-Si may change with a distance. On the basis of the presented data one can assume the macroscopic fluctuations of por-Si band gap produce the spatial separation of nonequilibrium charge carriers and photovoltage generation in the por-Si bulk.

Spatial non-uniformity of por-Si layer also should up in studying the PC spectra as a function of reverse bias voltage (Fig. 2). It should be noted that when the bias voltage was applied in forward direction the photocurrent value was considerably small as compared with the correspondent value under reverse bias voltage. The reverse bias condition corresponds to the case in which a positive potential is applied to the porous silicon surface. Such results can be explained by the presence of the potential barrier located between por-Si and silicon substrate.

The existence of potential barrier in studied structures is confirmed by current-voltage measurements. So, the cells exhibit a rectifying junction behaviour (Fig. 3). The inset shows the current-voltage characteristics under reverse bias condition (i) in the dark, and (ii) under 8$\mathrm{mW} / \mathrm{cm}^{2}$ tungsten lamp illumination. The ratio between the current in forward and reverse direction is $3.3 \times 10^{3}$, measured with $10 \mathrm{~V}$ across the sample. As is followed from the analysis, the current-voltage characteristic under forward bias condition is described by the equation $J=a U^{2}$, where $a=40.7 \mu \mathrm{A} / \mathrm{V}^{2}$.

Both the prevailing of PC component caused by porSi layer (in the region $h v>1.3 \mathrm{eV}$ ) and the observation of rectified current-voltage characteristics indicates to presence of two mechanisms of changing the current value under illumination. There are the increasing the por-Si conductivity and decreasing the potential barrier height of por-Si/c-Si heterojunction. Obviously, the last mechanism is predominant in the long-wave region where the porous silicon layer is transparent. The shape of the PC spectra in the spectral region $h v>1.3 \mathrm{eV}$ as well as the $\mathrm{PV}$ spectrum is caused by bulk properties of por-Si.

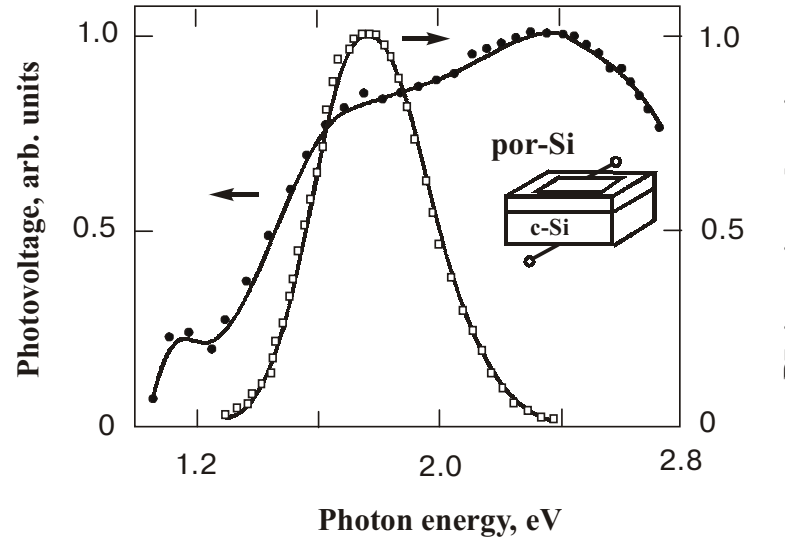

Fig. 1. The spectral dependencies of the photovoltage and photoluminescence for the por-Si/c-Si structure.

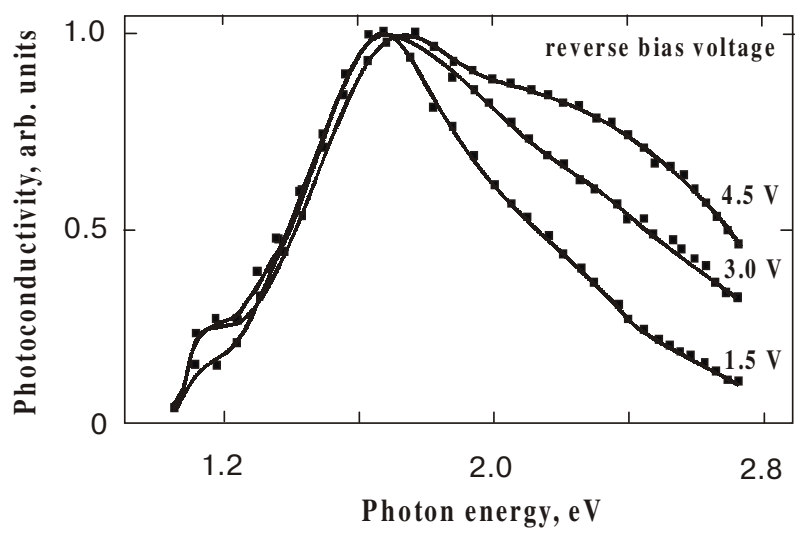

Fig. 2. The photoconductivity spectra of por-Si/c-Si structure as a function of reverse bias voltage.

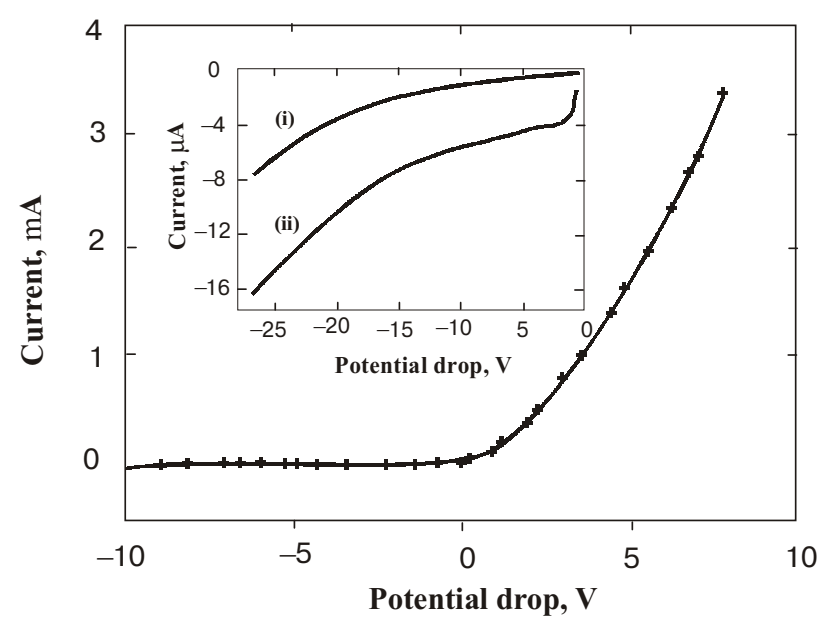

Fig. 3. The current-voltage characteristic of por-Si/c-Si structure. The inset shows the current-voltage characteristics under reverse bias voltage when a positive potential is applied to porSi surface (i) dark current, and (ii) current under $8-\mathrm{mW} / \mathrm{cm}^{2}$ tungsten lamp illumination. 


\section{O.V. Vakulenko, S.V. Kondratenko: Photovoltage and photocurrent spectroscopy of ...}

Let us focus on comparison of PC and PV spectrum shapes. The essential difference between photovoltage and photoconductivity spectral dependencies is taken place in the short-wave region. Namely, the PV spectral dependence has two components caused by the por-Si bulk (see Fig. 1), while the only one of them (long-wave) component is observed at studying of photoconductivity. Such particularity is a consequence of por-Si heterogeneity, i.e. difference conductivity and band gap of crystallites and silicon compounds $\mathrm{SiO}_{\mathrm{x}} \mathrm{H}_{\mathrm{y}}$.

As following from photovoltage and photocurrent spectroscopy the adsorption of excited quanta by silicon crystalline and silicon compounds $\mathrm{SiO}_{x} \mathrm{H}_{y}$ observes in different spectral ranges. Effect of hydrogen content on optical properties of por-Si is known. Authors [30] studied the influence of vacuum annealing on the absorption spectra shape of the free-standing porous silicon films. It was found [30] that vacuum annealing leads to hydrogen effusion and long-wave shift of the absorption edge by approximately to $0.5 \mathrm{eV}$ (from 1.7 to $1.2 \mathrm{eV}$ ). Therewith, the absorption edge of the annealed sample was close to the optical absorption edge of crystalline silicon.

In our case the nonequilibrium charge carries generated in $\mathrm{SiO}_{\mathrm{x}} \mathrm{H}_{\mathrm{y}}$ exhibit in photovoltage and don't contribute to photocurrent. To explain such features is possible by means of following arguments. It is generally accepted that in such composite system as porous silicon a charge will move along special path with the lowest resistance both on a grain-like skeleton of silicon crystallites and between the grains [25]. One can assume that the amorphous matrix of por-Si is practically excluded from the conduction process due to its less conductance in studied samples. Consequently, the nonequilibrium charge carriers generated in $\mathrm{SiO}_{\mathrm{x}} \mathrm{H}_{\mathrm{y}}$ do not contribute to the photocurrent value and the shape of the photoconductivity spectrum in the region $h v>1.3 \mathrm{eV}$ is caused mainly by band gap of silicon crystallites.

So, the shape of the photoconductivity spectra in the range from 1.3 to $2.7 \mathrm{eV}$ is caused mainly by adsorption of light quanta in silicon crystallites. The observation of photoconductivity in such wide spectral range indicates to existence of crystallite assembly with different band gap. The spatial distribution of crystallite sizes is most likely in direction perpendicular to the top surface of por-Si layer $[17,18,24]$. To explain the effect of reverse bias voltage on PC spectrum shape (see Fig. 2) is possible taking into account the proposed assumption. The relative contribution of crystallites located closer to the top surface of por-Si layer is growing at increasing of reverse bias voltage. Such crystallites have less size and greater band gap. As a consequence, the non-uniform increase of the photocurrent is observed in different spectral region while the enhancement at increasing of bias voltage is greater in the short-wave region. However, the additional experiments are necessary to prove finally the spatial dispersion of size and band gap width of silicon crystallites.
The studied samples exhibit an intense photoluminescence too. The photoluminescence spectra were taken at room temperature using $\mathrm{N}_{2}$ laser $(337 \mathrm{~nm})$. The spectral dependence for a typical porous silicon sample is shown in the Fig. 1. The maximum of the photoluminescence spectrum is at $1.77 \mathrm{eV}$ and the band width is about $0.44 \mathrm{eV}$. It should be pointed out that the photoluminescence band is observed in the same spectral range as the component of the photovoltage or photoconductivity spectra caused by por-Si. One can therefore conclude that the spectral position of the photoluminescence band in this case is determined by band gap width of silicon crystallites.

In conclusion, we have studied photovoltage, photoconductivity and photovoltage spectra in por-Si/c-Si samples to understand the physical reasons which cause the shape of the PC and PV spectra in por-Si and to clear up the influence of its composite structure on nonequilibrium processes. The band gap fluctuation of por-Si is revealed by photocurrent and photovoltage spectroscopy. Both two photovoltage and one photoconductivity component caused by por-Si material is observed. Such results are explained by composite por-Si structure which leads to the different band gap of crystallites and silicon compounds $\mathrm{SiO}_{\mathrm{x}} \mathrm{H}_{\mathrm{y}}$ on its surface.

\section{References}

1. L.T.Canham. Appl. Phys. Lett. 57 1016, (1990).

2. T. Ozaki, M. Araki, S. Yoshimura, H. Koyama, N. Koshida. J.Appl.Phys. 76, 1986 (1994).

3. S.V. Svechnikov, E.B. Kaganovich and E.G. Manoilov. Semiconductors Physics, Quantum Electronics \& Optoelectronics 1, 13 (1998).

4. Koshida N. and Koyama M. Appl. Phys. Lett. 60, 347 (1992).

5. C.Baratto, E.Comini, G.Faglia, G.Sberveglieri, G.Di Francia, F.De Filippo, V.La Ferrara, L. Quercia, L. Lancellotti. Sensors and Actuators A. 65, 257 (2000).

6. S.M.Prokes. J. Appl. Phys. 73, 407 (1993).

7. V. Petrova-Koch, T. Muschik, A. Kux, B.K. Meyer, F. Koch, and V. Lehmann, Appl.Phys.Lett. 61, 943 (1992).

8. Y. Kanemitsu, Phys.Rev.B 49, 16845 (1994).

9. G.Cullis, L.T.Canham, and P.D.G.Calcott, J.Appl.Phys. 82, 909 (1997).

10. O.V.Vakulenko, S.V. Kondratenko, and B.M. Shutov. Ukrainian Journal of Physics 44, 1465 (1999).

11. V.Yu.Timoshenko, E.A. Konstantinova, Th. Dittrich, Semiconductors 32, 613 (1998).

12. L.V.Belyakov, D.N.Goryachev, O.M. Sreselly, I.D. Yaroshetsky, Semiconductors 27, 1371 (1993).

13. E.V.Astrova, A.A. Lebedev, A.D. Remenyuk, V.Yu. Rud“,Yu.V. Rud“, Semiconductors 31, 159 (1997).

14. Feng Yan, Xi-Mao Bao and Ting Gao, Sol. State Comm. 91, 341 (1994).

15. R. Sedlacik, F. Karel, J. Oswald, A.Fejfar, I. Pelant, J. Kocka, Thin Sol. Films. 255, 269 (1995).

16. L. Burstein, Y. Shapira, J. Partee, J. Shinar, Y. Lubianiker, I. Balberg, Phys.Rev.B. 55, 1930 (1997).

17. J.D.Moreno, F.Agullo-Rueda, E.Montoya, M.L.Marcos, J.Gonzalez-Velasco, R.Guerrero-Lemus, J.M.MartinezDuart, Appl.Phys.Lett. 71, 2166 (1997).

18. S. Manotas, F. Agully-Rueda, J. D. Moreno, R. J. MartynPalma, R. Guerrero-Lemus, J. M. Martynez-Duart, Appl. Phys. Lett. 75, 977 (1999). 
O.V. Vakulenko, S.V. Kondratenko: Photovoltage and photocurrent spectroscopy of ...

19. D. Papadimitriou, J. Bitsakis, J.M. Lypez-Villegas, J. Samitier, J.R. Morante, Thin.Sol.Films. 349, 293 (1999).

20. J.D.Moreno, F.Agullo-Rueda, E.Montoya, M.L.Marcos, J.Gonzalez-Velasco, R.Guerrero-Lemus, J.M.MartinezDuart, Phys. Stat. Sol.(a) 182, 245 (2000).

21. Yu.A. Vashpanov, Pisma v Gurnal Technicheskoi Physici (in Russian) 23, 77 (1997).

22. A.B. Matveeva, E.A. Konstantinova, V.Yu. Timoshenko, P.K. Kashkarov, Semiconductors 29, 2180 (1995).

23. O.V.Vakulenko, S.V. Kondratenko, B.K.Serdega, Semiconductors Physics, Quantum Electronics \& Optoelectronics 4, 159 (2001).
24. V.M. Aroutionian, Phys.Stat.Sol.(a) 165, 105 (1998).

25. M.Ben-Chorin, F.Moller, and F.Koch, Phys.Rev.B 49, 2981 (1994).

26. V.Yu. Rud“,Yu.V. Rud“, Semiconductors 31, 245 (1997).

27. F.P.Romstad and E.Veje, Phys.Rev.B 55, 5220 (1997).

28. A.G. Milnes and D.L. Feucht, Heterojunctions and metalsemiconductor junctions (Academic press, New York and London, 1972).

29. S.M. Prokes, J.A. Freitas Jr. and P.S. Searson, Appl.Phys. Lett. 60, 3295 (1992).

30. L.A. Balagurov, D.G. Yarkin, E.A. Petrova, A.F. Orlov, S.N. Karyagin, Appl.Phys.Lett. 69, 2852 (1996). 